\title{
ELECCIONES AL PARLAMENTO VASCO
}

E1 9 de marzo de 1980 tuvieron lugar las elecciones al Parlamento vasco. Los resultados electorales remodelan, en buena medida, el mapa electoral configurado en el País Vasco sobre la base de las elecciones generales del 1 de marzo de 1979. Sucintamente los aspectos más relevantes han sido los siguientes:

\section{EL ascenso DE Los Nacionalistas y La CONSOLIDACION DE LAS POSICIONES ABERTZALES}

El Partido Nacionalista Vasco ha sido, sin duda, el gran triunfador de estas elecciones. Con una campaña basada, por una parte, en la defensa del Estatuto de Autonomía y de su aplicación integra e inmediata y, por otra, en la necesidad de una mayoría fuerte para resolver la fuerte crisis económica, ha sabido hacer converger hacia sus posiciones, por distintos motivos, a una gran parte del electorado vasco.

El PNV aumentó 75.983 votos en relación a los obtenidos el 1 de marzo de 1979 (ver cuadro núm. 1). Desplaza a UCD como primera fuerza política en Alava con lo que se constituye en el primer partido político de las tres provincias vascas. Los 350.286 votos (ver cuadro núm. 2) que obtienen representan un 22 por 100 del total del electorado vasco y, si bien los veinticinco diputados que le han sido asignados no le permiten obtener la mayoría absoluta, le concede una gran capacidad de maniobra en el Parlamento, sobre todo en caso de persistir la negativa de Herri Batasuna de acudir al mismo.

Por lo que hace referencia a las posiciones abertzales, la coalición Herri Batasuna consolida su fuerza electoral. No registra un fuerte aumento de votos - los votos obtenidos en esta ocasión son sólo 3.477 más que en marzo de 1979 (ver cuadro núm. 2)-, pero la vertiginosa caída de UCD y PSOE le ayudan a convertirse en la segunda fuerza política y alcanzan una representación parlamentaria de once diputados (ver cuadro núm. 2).

Por su parte, Euzkadiko Ezquerra, que mantiene una progresión ascendente muy regular desde las elecciones del 15 de junio de 1977, aumenta en relación a las últimas elecciones generales 9.966 votos (ver cuadro núm. 1). Si bien su representación parlamentaria es igual a la alcanzada por UCD, esto es, seis diputados (ver cuadro núm. 2), en cuanto al número de votos se pasa a ser la cuarta fuerza política. 
Los escaños nacionalistas y abertzales constituyen, por tanto, mayoría absoluta en el Parlamento. Contando PNV, HB y EE con un total de 42 escaños frente a los 18 que suman los partidos de carácter estatal.

\section{EL FUERTE DESCENSO DE LOS PARTIDOS DE AMBITO ESTATAL}

La pérdida de votos de la mayoría de los partidos de carácter estatal viene siendo en el País Vasco una constante desde las primeras elecciones generales.

Unión de Centro Democrático es de entre estos partidos el que ha sufrido un mayor deterioro de su fuerza electoral en estas elecciones. El partido del Gobierno cede el primer lugar que ocupara, desde el 15 de junio de 1977, en la provincia de Alava al PNV. Con una pérdida de 90.000 votos — por relación a las elecciones del 1 de marzo de 1979 (ver cuadro núm. 1) - en el conjunto de las tres pro-

CUADro NúM. 1

FUERZA ELECTORAL DE LOS PARTIDOS

DEL PAIS VASCO QUE HAN ALCANZADO REPRESENTACION PARLAMENTARIA

(núm. total de votos)

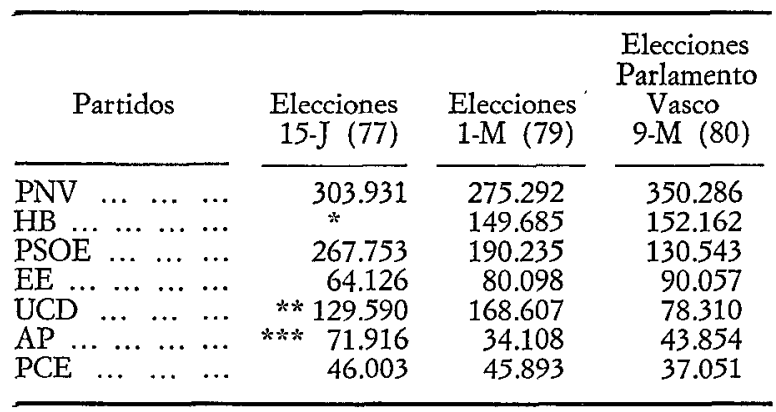

Fuentes: Diario El Pais, 11 de marzo de 1980. Datos del Ministerio del Interior.

* HB no existía en 1977

** UCD no se presentó en Guipúzcoa en 1977.

*** AP se presentó en 1977 en Guipúzcoa cón el nombre de Guipúzcoa Unída.

vincias vascas pasa a ocupar, ahora, el quinto lugar entre las fuerzas políticas del País Vasco y sus votos alcanzan a representar a un 5 por 100 del censo electoral del mismo. Un análisis riguroso de donde hayan ido a parar los votos perdidos por UCD traspasan los límites de esta nota, no obstante ello queremos dejar apuntadas algunas hipótesis al respecto.

En primer lugar, parece tener bastante credibilidad la suposición de que Alianza Popular se haya visto beneficiada de la pérdida de votos de UCD. AP es el único partido de ámbito estatal que mejora sus posiciones - mejora a la que probablemente no es ajena la progresiva ideologización y consecuente polarización política desarrollada en el País Vasco- ganando cerca de 10.000 votos en relación al 1 de marzo de 1979 (ver cuadro núm. 1). El PNV parece haber resultado el otro partido más claramente beneficiado por el descenso del número de votantes de UCD. Ello se justificaría sobre la base de la confianza que este partido ha intentado, y parece haber conseguido, irradiar en el empresariado vasco o cuando menos a sectores importantes del mismo. Por último, entre los abstencionistas, que 
CUAdRo NúM. 2

ELECCIONES AL PARLAMENTO VASCO (9-III-1980)

\begin{tabular}{|c|c|c|c|c|c|c|c|c|}
\hline \multirow[b]{3}{*}{ Candidaturas } & \multicolumn{6}{|c|}{ PROVINCIAS } & & \\
\hline & \multicolumn{2}{|c|}{ Alava } & \multicolumn{2}{|c|}{ Guipúzcoa } & \multicolumn{2}{|c|}{ Vizcaya } & \multicolumn{2}{|c|}{ Total } \\
\hline & Votos & Escaños & Votos & Escaños & Votos & Escaños & Votos & Escaños \\
\hline $\begin{array}{lllllll}\mathrm{LKI} & \ldots & \ldots & \ldots & \ldots & \ldots & \ldots\end{array}$ & 437 & & 2.092 & & 2.645 & & 5.174 & \\
\hline $\begin{array}{lllllll}\mathrm{EE} & \ldots & \ldots & \ldots & \ldots & \ldots & \ldots\end{array}$ & 9.652 & 2 & 40.137 & 3 & 40.268 & 1 & 90.057 & 6 \\
\hline $\begin{array}{lllll}\text { PSE-PSOE } & \ldots & \ldots & \ldots & \ldots\end{array}$ & 14.694 & 3 & 41.100 & 3 & 74.749 & 3 & 130.543 & 9 \\
\hline $\begin{array}{lllll}\text { PCE-EPK } & \ldots & \ldots & \ldots & \ldots\end{array}$ & 3.172 & & 9.016 & & 24.863 & 1 & 37.051 & 1 \\
\hline $\begin{array}{lllllll}\text { CUC } & \ldots & \ldots & \ldots & \ldots & \ldots & \ldots\end{array}$ & 158 & & 544 & & 1.350 & & 2.052 & \\
\hline $\begin{array}{lllllll}\mathrm{UCD} & \ldots & \ldots & \ldots & \ldots & \ldots & \ldots\end{array}$ & 20.616 & 4 & 22.954 & 1 & 34.740 & 1 & 78.310 & 6 \\
\hline $\begin{array}{llllll}\operatorname{EMK} & \ldots & \ldots & \ldots & \ldots & \ldots\end{array}$ & 768 & & 4.508 & & 5.677 & & 10.953 & \\
\hline $\begin{array}{lllllll}\text { PNV } & \ldots & \ldots & \ldots & \ldots & \ldots & \ldots\end{array}$ & 31.640 & 7 & 111.274 & 9 & 207.369 & 9 & 350.286 & 25 \\
\hline $\begin{array}{lllllll}\mathrm{EKA} & \ldots & \ldots & \ldots & \ldots & \ldots & \ldots\end{array}$ & 465 & & 1.275 & & 690 & & 2.430 & \\
\hline $\begin{array}{lllllll}\mathrm{HB} & \ldots & \ldots & \ldots & \ldots & \ldots & \ldots\end{array}$ & 14.804 & 3 & 52.294 & 4 & 85.064 & 4 & 152.162 & 11 \\
\hline $\begin{array}{lllll}\text { ORT-PTE } & \ldots & \ldots & \ldots & \ldots\end{array}$ & 734 & & 1.316 & & 1.425 & & 3.475 & \\
\hline 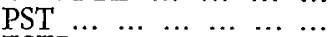 & - & & - & & 2.105 & & 2.105 & \\
\hline 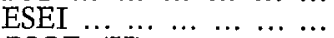 & 1.412 & & 3.559 & & 1.333 & & 6.304 & \\
\hline $\begin{array}{lllll}\text { PSOE }(\mathrm{H}) & \ldots & \ldots & \ldots & \ldots\end{array}$ & - & & - & & 2.808 & & 2.808 & \\
\hline $\begin{array}{lllll}\text { FE-JONS } & \ldots & \ldots & \ldots & \ldots\end{array}$ & - & & - & & 1.466 & & 1.466 & \\
\hline $\begin{array}{lllllll}\mathrm{AP} & \ldots & \ldots & \ldots & \ldots & \ldots & \ldots\end{array}$ & 5.974 & 1 & 7.980 & & 29.900 & 1 & 43.854 & 2 \\
\hline * Total votos $\ldots \ldots \ldots$ & 106.136 & $59,01 \%$ & 301.475 & $57,94 \%$ & 524.514 & $59,30 \%$ & & \\
\hline Censo electoral $\ldots \ldots \ldots$ & 179.844 & & 520.316 & & 884.417 & & & \\
\hline ** Votos blancos $\ldots \quad \ldots$ & 603 & $0,56 \%$ & 795 & $0,26 \%$ & 2.192 & $0,41 \%$ & & \\
\hline$* *$ Votos nulos $\ldots \ldots \ldots$ & 980 & $0,92 \%$ & 2.632 & $0,87 \%$ & 5.870 & $7,11 \%$ & & \\
\hline$*$ Abstenciones $\ldots \ldots$ & 73.704 & $40,98 \%$ & 218.841 & $42,05 \%$ & 359.903 & $40,69 \%$ & & \\
\hline
\end{tabular}

* Porcentaje calculado sobre censo electoral.
*** Porcentaje calculado sobre total de votos.

Fuenre: Datos facilitados por el Ministerio del Interior.

LKI: Liga Komunista Ikaulzailea; EE: Euskadiko Ezkerra; PSE-PSOE: Partido Socialista de Euskadi; PCE-EPK: Partido Comunista de Euskadi-Euskadiko Partidu Komunista; CUC: Coalición de Unidad Comunista; UCD: Unión de Centro Democrático; EMK: Movimiento Comunista de Euskadi; PNV: Partido Nacionalista Vasco; EKA: Partido Carlista; HB: Agrupación electoral Herri Batasuna; ORT-PTE: Partido de los Trabajadores de Euskadi; PST: Partido Socialista de los Trabajadores; ESEI: Unificación de Socialistas de Euskadi; PSOE (H): Partido Socialista Obrero Español (Histórico); FE-JONS: Falange Española de las JONS; AP: Alianza Popular. 
constituyen el 41 por 100 de los electores, podría encontrarse buena parte del electorado perdido por UCD.

El PSOE ha sido, aunque en menor medida, el otro gran perdedor de estas elecciones. Pierde alrededor de 60.000 votos desde las elecciones de marzo de 1979 (ver cuadro núm. 1) - si bien esta cifra se ve extraordinariamente aumentada si se compara con los resultados que obtuviera el 15 de junio de 1977 - y consigue nueve diputados. La abstención podría haberle afectado por los mismos motivos por los que se habría visto afectada UCD, es decir, en tanto aquélla hubiere incidido fuertemente en la población inmigrante.

El Partido Comunista, por su parte, sufre una pérdida de 8.842 votos (ver cuadro núm. 1) y consigue un diputado.

\section{LA VICTORIA DE LAS POSICIONES MAS CONSERVADORAS EN EL ORDEN SOCIAL}

Cuando se aborda el análisis de los resultados electorales en el País Vasco y sus consecuencias más inmediatas a nivel parlamentario, suele obviarse, o dejarse en un segundo plano, en significación desde la perspectiva de las posiciones económicas y sociales que defienden los partidos de alcanzada representación en el Parlamento. Sin duda ello viene, en buena medida, justificado por el papel determinante que ha venido jugando el problema nacional en la conflictiva situación del Pá́s Vasco. El tratamiento del citado problema se ha convertido en el punto de referencia, fundamental, para las alianzas o el enfrentamiento político, elevándose por encima de otras diferencias o convergencias entre los partidos. Sin embargo, y toda vez que el Parlamento Vasco va a contar competencias en el orden social y económico, nos parece oportuno señalar cuáles han sido los resultados electorales desde la perspectiva apuntada.

E1 balance aparece claramente favorable a las proposiciones conservadoras. Los partidos que a estos efectos se encuentran situados del centro a la derecha del espectro político han obtenido un total de 33 parlamentarios. Incluso sin contar con Alianza Popular, el PNV y UCD - que tienen, sin duda, un mayor grado de convergencia en los aspectos mencionados- cuentan con la mayoría absoluta de la Cámara.

CuAdro Núm. 3

ELECCIONES AL PARLAMENTO VASCO (9-III-1980)

GUIPUZCOA: ABSTENCION EN LOS MUNICIPIOS DE MAS DE 20.000 HABITANTES

\begin{tabular}{|c|c|c|}
\hline Municipios & $\begin{array}{l}\text { Censo } \\
\text { electoral }\end{array}$ & $\begin{array}{l}\% \text { abstención } \\
\text { sobre censo }\end{array}$ \\
\hline $\begin{array}{llllllll}\text { Eibar } & \ldots & \ldots & \ldots & \ldots & \ldots & \ldots & \ldots \\
\text { Hernani } & \ldots & \ldots & \ldots & \ldots & \ldots & \ldots \\
\text { Irún } \ldots & \ldots & \ldots & \ldots & \ldots & \ldots & \ldots & \ldots \\
\text { Mondragón } & \ldots & \ldots & \ldots & \ldots & \ldots & \ldots \\
\text { Pasajes } & \ldots & \ldots & \ldots & \ldots & \ldots & \ldots & \ldots \\
\text { Rentería } & \ldots & \ldots & \ldots & \ldots & \ldots & \ldots \\
\text { San Sebastián } & \ldots & \ldots & \ldots & \ldots \\
\end{array}$ & $\begin{array}{r}29.076 \\
20.904 \\
38.400 \\
18.786 \\
16.977 \\
33.139 \\
136.089\end{array}$ & $\begin{array}{l}41,21 \\
44,58 \\
42,65 \\
41,99 \\
49,52 \\
45,92 \\
44,72\end{array}$ \\
\hline $\begin{array}{lllll} & \ldots & \ldots & \ldots & \ldots\end{array}$ & 520.316 & 42,05 \\
\hline
\end{tabular}

FuENTE: Datos ofrecidos por el Ministerio del Interior. 
La izquierda del abanico político - PSOE, PC, EE y HB- han alcanzado una menor representación parlamentaria, sumando entre ellos 27 diputados, aunque estas distancias se ven algo atenuadas si atendemos a las diferencias en votos obtenidas por uno y otro bloque de partidos.

\section{EL AUMENTO DE LA ABSTENCION}

El índice medio de abstención en las tres provincias vascas se eleva en un 6 por 100 por encima del alcanzado en las elecciones legislativas de 1979 .

La abstención en la mayoría de los núcleos industriales -fundamentalmente en Guipúzcoa y Vizcaya (ver cuadros núm. 3 y 4)- supera la media alcanzada en

CUADRO NÚM. 4

ELECCIONES AL PARLAMENTO VASCO (9-III-1980)

VIZCAYA: ABSTENCION EN LOS MUNICIPIOS DE MAS DE 20.000 HABITANTES

\begin{tabular}{|c|c|c|}
\hline Municipios & $\begin{array}{l}\text { Censo } \\
\text { electoral }\end{array}$ & $\begin{array}{c}\% \text { abstención } \\
\text { sobre censo }\end{array}$ \\
\hline 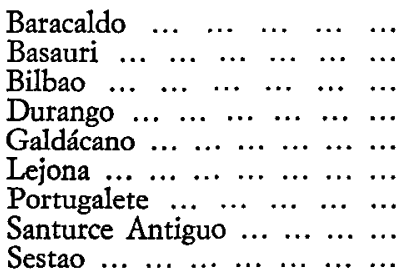 & $\begin{array}{r}89.744 \\
38.082 \\
336.708 \\
18.971 \\
17.945 \\
13.804 \\
42.985 \\
37.910 \\
30.475\end{array}$ & $\begin{array}{l}40,59 \\
46,42 \\
41,05 \\
37,63 \\
39,64 \\
42,62 \\
47,66 \\
46,06 \\
42,70\end{array}$ \\
\hline $\begin{array}{lllllll}\text { Vizcaya } & \ldots & \ldots & \ldots & \ldots & \ldots & \ldots\end{array}$ & 884.417 & 40,69 \\
\hline
\end{tabular}

Fuente: Datos ofrecidos por el Ministerio del Interior.

sus respectivas provincias. Podría parecer aventurado afirmar que los sectores abstencionistas hayan sido, prioritariamente, los constituidos por la población inmigrante que en su gran mayoría vive en las zonas industriales. De la misma manera no se puede afirmar taxativamente que la pérdida de votos del PSOE - que tradicionalmente obtiene sus mejores resultados en aquellos municipios- y UCD se explica en base a la abstención de este sector del electorado.

Las inferencias a partir de los datos aquí ofrecidos sólo pueden constituir hipótesis para un trabajo más extenso.

\section{A MODO DE CONCLUSION}

Sobre la base de los datos hasta aquí ofrecidos se puede apuntar que la vida parlamentaria vasca se va a encontrar configurada por el desarrollo de dos ejes y por la posible interacción entre éstos. El primero de los vectores es el constituido por la problemática nacional. En torno al mismo, las alianzas las tendría que buscar el PNV - partido que juega el papel de clave de arco- en los partidos 
abertzales con los que conseguiría la mayoría absoluta en el Parlamento, sin dejar por ello de contar, a esos efectos, con los partidos de izquierda de ámbito estatal que, en ciertos casos, podrían reforzar, en buena medida, sus posiciones.

El segundo de los vectores estaría constituido por las actitudes políticas ante la problemática social. En relación a este segundo aspecto, el PNV podría tener que recabar la alianza de UCD para obtener la mayoría absoluta de la Cámara.

No es necesario decir que el futuro del Parlamento vasco va a estar condicionado por la vertebración de la política de la mayoría parlamentaria en torno a uno u otro de los ejes apuntados.

Pilar del Castillo Vera

Universidad Nacional de Educación a Distancia 